\title{
Enhancement of UAV-Aerial Images Using Weighted Differential Evolution Algorithm
}

\author{
Ahmet Emin Karkınlı"1, Abdüsselam Kesikoğlu ${ }^{2}$ \\ ${ }^{*}$ Department of Geomatic Engineering, Niğde Ömer Halisdemir University, Niğde, TÜRKIYE \\ ${ }^{2}$ Tomarza MA Vocational School, Kayseri University, Kayseri, TÜRKIYE
}

\section{Article Info}

Volume 7, Issue 2

Page Number: 196-206

Publication Issue :

March-April-2021

\section{Article History}

Accepted : 01 April 2021

Published : 03 April 2021

\section{ABSTRACT}

Depending on technological developments, the use of Unmanned Aerial Vehicles (UAVs) is increasing day by day and is a valuable source of data for different applications. Generally, low-cost and lightweight non-metric digital cameras are used in UAV systems. During the data collection phase, exposure parameters such as camera shutter speed, aperture value, ISO value, and various weather and light conditions have significant effects on image quality. Image enhancement methods can be used to increase image quality in accordance with the desired purpose. In this study, image enhancement is considered as an optimization problem and Weighted Differential Evolution (WDE) Algorithm is used to solve it. The image quality is enhanced by using an objective function in which performance measures of entropy value, sum of edge density and number of edge pixel are maximized. In the proposed color image enhancement method, aerial images defined in RGB color space are transformed into HSV color space images. the brightness component $(\mathrm{V})$ of HSV color space is modified for image improvement with WDE algorithm. The performance of the proposed method has been compared with other existing techniques such as histogram equalization, linear contrast stretching and evolutionary computing-based image enhancement method like Artificial Bee Colony (ABC) Algorithm in terms of fitness value and image quality.

Keywords : Image Enhancement, Evolutionary Algorithm, Weighted Differential Evolution (WDE) Algorithm, Log Transform Function

\section{INTRODUCTION}

The fact that unmanned aerial vehicles can produce a wide range of cheap and fast solutions is considered suitable for many potential application areas in different disciplines [1]. UAVs are also used for control, surveillance, mapping and 3D modelling purposes such as 3D documentation of archaeological sites and buildings [2], post-disaster response and mapping [3], monitoring environmental soil and water changes [4], deformation tracking [5], volume calculations for excavation areas [6] and recording natural resources [7]. 
UAVs with limited payload capacity cannot carry high-end image capture equipment, so non-metric cameras with small sensor and limited lens quality can be used [8]. Besides the quality of the image capture equipment, a variety of reasons such as the inability to selecting ideal exposure parameters, weather and light conditions and hardware stability affects the contrast and clarity of the obtained images [9]. As a result of these conditions, high-quality images cannot be captured and the images need to be improved. Image enhancement algorithms are clearly required for a properly usable image.

The aim of image enhancement is to increase the perceptibility or interpretability of images for visual analysis or to provide a "better" input image to other automatic image processing techniques [10]. Image enhancement techniques can be divided into frequency domain and spatial domain categories. The frequency domain includes operating techniques in transforming the image frequency, while the spatial domain technique improves the contrast and brightness of the image pixels [11]. Some of the wellknown spatial domain image enhancement techniques are histogram equalization [12], contrast stretching [13], intensity transformation functions [14-16], intensity level slicing, etc. As a result of these enhancements, the pixel value (densities) of the output image is updated by changing the brightness, contrast, or gray level distribution of the input image.

The ability of heuristic optimization methods to converge to global optimum provides advantages over classical methods in solving different problems. For image enhancement in the spatial domain, transformation functions are used that generate a new intensity value for each pixel of the original image. Optimum parameters for the objective functions that will minimize or maximize the desired features for image enhancement can be achieved very successfully with heuristic algorithms [17]. In [14], a cost function based on entropy value, sum of edge densities and increasing the number of edges is defined and three performance measures are maximized with Particle Swarm Optimization (PSO). In different image enhancement studies, Genetic Algorithm (GA) [18], Differential Evolution Algorithm (DE) [19], Dragonfly Algorithm (DA) [20], Ant Colony Optimization (ACO) [21], Cuckoo Search Algorithm (CS), Backtracking Search Algorithm (BSA) [17] and Differential Search Algorithm (DSA) [22] were used.

Weighted Differential Evolution Algorithm (WDE) is a new iterative, evolutionary search algorithm developed to solve real valued numerical optimization problems [23]. The structure of the WDE algorithm, which does not require preadjustment and has no parameters, makes it easy to use in different problems [24]. WDE was preferred in this study because it gave successful results for different problems in comparison studies in the literature [25].

In the proposed color image enhancement method, aerial images defined in RGB color space are transformed into HSV color space images. Hue $(\mathrm{H})$ and saturation (S) components keep constant, the brightness component $(\mathrm{V})$ is modified for image improvement with WDE algorithm. Finally, it is converted to an image in RGB color space using the modified brightness component $(\mathrm{V})$ and the original Hue $(\mathrm{H})$ and Saturation $(\mathrm{S})$ components. The performance of the proposed method has been compared with other existing techniques such as histogram equalization, linear contrast stretching and evolutionary computing-based image enhancement method like Artificial Bee Colony (ABC) Algorithm in terms of fitness value and image quality.

The organization of this document is as follows. In Section 2 (Image Enhancement), Some approaches 
used for color image enhancement and enhancement function are given. In Section 3 (Heuristic Optimization Methods), The WDE and ABC algorithms used in the study are introduced. In Section 4 (Experimental Results and Discussion), The performance of the proposed method has been compared with different methods and the results have been shown.

\section{IMAGE ENHANCEMENT}

Different approaches can be applied in RGB color image enhancement. Image enhancement functions can be applied to each R, G, B component image independently [26]. In these methods, since each $R$, G, B image component is developed independently, the color components in each pixel may vary at a different rate. For this reason, color change may occur in the output image [27].

Another approach is to transform RGB color images into HSV, LHS, YIQ or etc. color spaces, and modify color components that contain only the desired properties [28, 29]. HSV color space is frequently used in the enhancement of color images. After transforming original images to HSV color images, Hue $(\mathrm{H})$ and saturation $(\mathrm{S})$ components keep constant, the brightness component $(\mathrm{V})$ is modified for image improvement. In literature some studies in which saturation (S) and brightness component (V) are changed together for image enhancement [30].

\section{A. Enhancement Function}

The basic equation of image enhancement is defined as in (1) [15]:

$$
g(x, y)=T[f(x, y)]
$$

Here $f(x, y)$ is the input image, $g(x, y)$ is the output image. $\mathrm{T}$ is the operation applied to $f$ defined in the neighbourhood of the $(x, y)$ point. Transformation function defined for adjusting contrast and brightness calculates a new pixel value for each pixel in the input image by processing the neighbourhoods of this pixel. The transformation function used is defined in (2).

$$
g(i, j)=K(i, j)[f(i, j)-c \times m(i, j)]+m(i, j)^{a}
$$

$m(i, j)$ is the local mean of the $(i, j)$ th pixel of the input image over an $\mathrm{n} \times \mathrm{n}$ window. Expression for local mean function is given as (3):

$$
m(i, j)=\frac{1}{n \times n} \sum_{x=0}^{n-1} \sum_{y=0}^{n-1} f(x, y)
$$

$K(i, j)$ is known as the enhancement function that considers both local and global information. This enhancement function is calculated according to (4):

$$
K(i, j)=\frac{k \cdot D}{\sigma(i, j)+b}
$$

Where " $\mathrm{D}$ " is the global mean value calculated for the entire image area and expression is calculated according to (5):

$$
D=\frac{1}{M \times N} \sum_{i=0}^{M-1} \sum_{j=0}^{N-1} f(i, j)
$$

$\sigma(i, j)$ is the local standard deviation. This expression is calculated according to (6):

$$
\sigma(i, j)=\sqrt{\frac{1}{n \times n} \sum_{x=0}^{n-1} \sum_{y=0}^{n-1}(f(x, y)-m(i, j))^{2}}
$$

Thus, the transformation function looks like (7):

$$
g(i, j)=\frac{k \cdot D}{\sigma(i, j)+b}[f(i, j)-c \times m(i, j)]+m(i, j)^{a}
$$

By this transformation (7), contrast of the image is stretched considering local mean as the centre of stretch. Four parameters are used in the transformation function, namely a, b, c, and k. In the proposed method, the WDE algorithm is applied to optimize these 4 parameter values of the transformation function.

\section{B. Enhancement Objective Function}

Objective evaluation criterion was used to measure the quality of the images improved as a result of the 
proposed method. This evaluation criterion, $(x)$, which considers the entropy value of the images, the number of edge pixels and the density (sobel value) of the pixels, is expressed as follows (8) $[14,16,31]$ :

$$
F(Z)=\log (\log (E(I(Z)))) \times \frac{n_{-} \text {edges }(I(Z))}{M \times N} \times H(I(Z))
$$

$\mathrm{F}(\mathrm{Z})$ is the cost function. $\mathrm{I}(\mathrm{Z})$ indicates the original I image with the $\mathrm{T}$ transform applied according to (1). $E(I(Z))$ is the intensity of the edges detected with a Sobel edge detector, $\mathrm{n}$ _edges is the number of edge pixels as detected with the Sobel edge detector, E(I) is the sum of intensities of the edges included in the enhanced image and $H(I(Z))$ refers to the entropy value of the $\mathrm{I}(\mathrm{Z})$ image.

\section{III.HEURISTIC OPTIMIZATION METHODS}

The concept of optimization is expressed as finding the conditions that give the maximum and minimum of a function. One type of well-known Optimization algorithm is evolutionary algorithms, also called heuristic algorithms [32]. Due to their advantages such as heuristic algorithms, high convergence speed to solution, global search capability, low parameter precision, and ease of application, they are widely used for solving problems in many different fields [32-34].

\section{A. Weighted Differential Evolution (WDE) Algorithm}

The Weighted Differential Evolution (WDE) algorithm is a new variation of the Differential Evolution (DE) algorithm proposed by Çivicioğlu et al. in 2018. In this algorithm, a new mutation operator is defined that works with two population sets in each iteration [23].

In the algorithm, firstly, two populations are formed, the initial population $(\mathrm{P})$ and the sub-population (SubP) randomly generated in accordance with the normal distribution. Next, the $\mathrm{N}$-dimensional TempP, called the trial vector, is generated by (9) presented below using the remainder of the solution vectors (Prest) not selected in the previous step.

$$
\text { Temp } P=\sum\left(w \circ P_{\text {rest }}\right) \text { where }\left\{\begin{array}{c}
w=\frac{w_{i}^{*}}{\sum_{v}^{n} w_{i}^{*}} \times \Delta \\
w^{*}=K_{(N \times 1)}^{3}
\end{array}\right.
$$

Here, "o" is the Hadamard operator, $\Delta$ is a $(1 \times D)$ dimensional vector whose elements are equal to 1 , and $K(N \times 1)$ is a $(N \times 1)$ dimensional vector made up of random numbers.

In the WDE algorithm, a control parameter $\left(M_{(1: N, 1: D)}=0\right)$, which will be updated during the iterations, is defined based on the equations presented to (10):

$$
M_{\text {(indexJ) }}:=1 \text { where }\left\{\begin{array}{l}
J=V(1:\lceil K \times D\rceil) \\
V=\text { permute }(j 0)
\end{array}\right.
$$

Here, $K$ is calculated by (11):

$$
\text { If } \alpha<\beta \text { then } K=\kappa_{(1)}^{3} \text { else } K=\left(1-\kappa_{(1)}^{3}\right)
$$

Here, $\alpha, \beta$ and $\kappa$ are randomly uniform numbers between 0 and 1 , and the presented $\kappa$ elements define the size of this vector.

In the algorithm, a scale factor represented by $\mathrm{F}$ is defined and is calculated according to (12):

$$
\left\{\begin{array}{l}
F_{(1 \times D)}=\lambda_{(D)}^{3} \mid \text { If } \alpha^{l}<\beta^{l} \\
F_{(N \times D)}=\left(\lambda_{(N)}^{3} \times \Delta\right) \mid \text { Otherwise }
\end{array}\right\}
$$

Here $\lambda$ is a vector consisting of uniform random numbers between 0 and 1. Finally, the new population $(T)$ is produced according to (13):

$$
\begin{aligned}
& T=S u b P+F \times M \circ\left(T e m p P-S u b P_{(m)}\right) \\
& m=\text { permute }(i) \mid m \neq[1: N]
\end{aligned}
$$

Here it is defined as $i=1: N$ and $i \in Z^{+}$ 


\section{B. Artificial Bee Colony (ABC) Algorithm}

The artificial bee colony algorithm (ABC), inspired by the behaviours honey bees display to seek food, models the unique behaviour of bees [35]. There are three types of bees in a colony of ABC: employed, onlookers and scouts. The quality of the nectar source that the bees investigated depends on its proximity to the hive, the extract and fullness of the nectar. Food sources represent possible solutions. Employed bees search for nectar sources and carry the results to the hive. Onlookers bees search for nectar and select a source of nectar by watching the dance of employed bees. Scout bees are in charge of searching for new sources of nectar by going to new sources randomly. This process is low cost because it is done randomly [36].

In $\mathrm{ABC}$, employed bees do the research of the nectar source with (14).

$$
v_{i j}=x_{i j}+\phi_{i j}\left(x_{i j}-x_{k j}\right)
$$

Here, $\mathrm{N}$ is the number of nectar sources, where $k \in\{1,2,3, \ldots, N\}, j \in\{1,2,3, \ldots, K\}, \mathrm{K}$ is the problem size, $\mathrm{x}$ is the current nectar source and $\mathrm{v}$ is the newly determined nectar source.

When the bees have access to more efficient solutions / resources, they keep the new solution in their memory. When all employed bees have finished their search, they share the resource positions / possible solutions with the onlooker bees. Onlookers bees evaluate the amount of nectar and select new sources based on information from employed bees. The probability $p_{i}$ of each source " $i$ " selected is obtained by dividing the selected source by the fitness value obtained from the total sources as shown by $(15)[37,38]$ :

$$
p_{i}=\frac{f i t}{\sum_{n}^{N} f i t_{n}}
$$

\section{EXPERIMENTAL RESULTS AND DISCUSSION}

In experimental studies, aerial images obtained from UAV with two different cameras were used. Tests were carried out with RGB images at 5472x3648 resolution recorded with DJI FC6310R model camera and 4000x3000 resolution recorded with DJI FC330 model camera. The original images are shown in Figure 1:
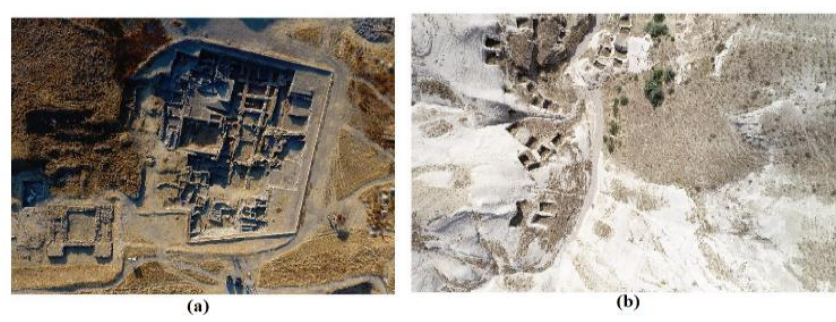

Figure 1: (a) Image I (DJI FC330 (4000x3000 res.)) (b) Image II (FC6310R camera (5472x3648 res.))

The objective evaluation criteria defined in (8) was used to measure the quality of the improved images. The parameters $\mathrm{a}, \mathrm{b}, \mathrm{c}$ and $\mathrm{k}$ in the transformation function have been optimized by WDE and $A B C$ algorithms. The lower and upper limit value ranges for these parameters were determined as $\mathrm{a} \epsilon[0,2], \mathrm{b} \epsilon$ $[0,1], c \in[0,0.5]$ and $\mathrm{k} \in[0,2]$, considering previous studies $[14,39,40]$. For heuristic optimizations, the population size was determined as 20 and the number of iterations as 250. The Weighted Differential Evolution (WDE) algorithm has no control parameters and is proposed for the solution of numerical optimization problems since control parameters are determined randomly [23]. The control parameters for the Artificial Bee Colony ABC algorithm are used as Limit $=N x D$ and Sizeofempoyedbee $=$ Sizeofcolony $/ 2$. Because heuristic algorithms use random starting values and random search processes, the algorithms were run 30 times 
and the average and standard deviation results of the obtained evaluation criteria are given in Table 1.

Table 1: Statistics for the solutions of WDE \& ABC

\begin{tabular}{|l|c|c|c|r|}
\hline \multirow{2}{*}{} & \multicolumn{2}{|c|}{ WDE } & \multicolumn{2}{c|}{ ABC } \\
\cline { 2 - 5 } Image & \multicolumn{2}{|c|}{ Fitness Value } & \multicolumn{2}{c|}{ Fitness Value } \\
\cline { 2 - 5 } & Mean & Std & Mean & \multicolumn{1}{c|}{ Std } \\
\hline & & & & $2.08 \mathrm{E}-$ \\
Image I & 0.6915881 & $5.00 \mathrm{E}-04$ & 0.6810607 & 03 \\
\hline Image & & & & $4.46 \mathrm{E}-$ \\
II & 0.6309580 & $3.78 \mathrm{E}-05$ & 0.6296347 & 03 \\
\hline
\end{tabular}

Histogram Equalization aims to redistribute the intensity of the image, to have a uniform distribution and to have approximately the same number of pixels for each brightness level [12]. Color histogram equalization can be achieved by converting a color image into HSV image and enhancing the Intensity (V) while preserving hue $(\mathrm{H})$ and saturation (S) components [41]. In Figure 2, the histogram distributions of the original image and the histogram equalized image are shown.
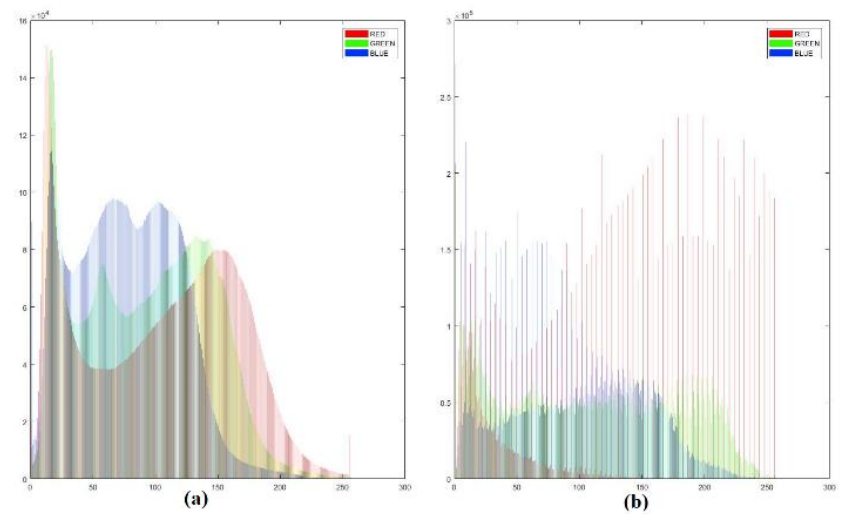

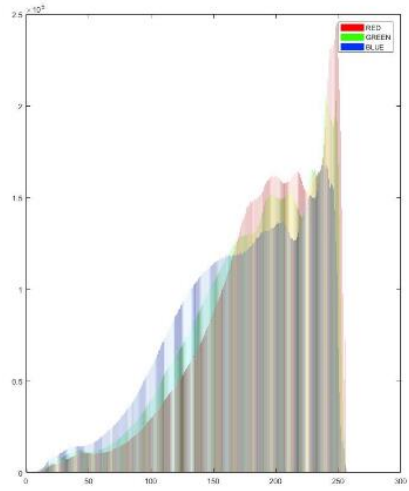

(c)

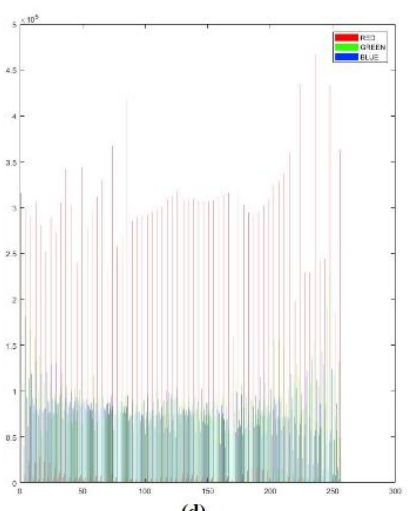

(d)
Figure 2: (a) Image I Histogram , (b) Image I Equalized Histogram, (c) Image II Histogram , (d) Image II Equalized Histogram

Histogram contrast stretching is a special case of histogram modification. It involves defining the lower and upper boundaries of images in which the histogram spans a narrow area and applying a transform to widen this range [42]. In this study, the lower and upper limits for histogram stretching were determined based on the image standard deviation and the mean value. In Figure 3, the histogram distributions of the original image and the histogram contrast stretched image are shown.

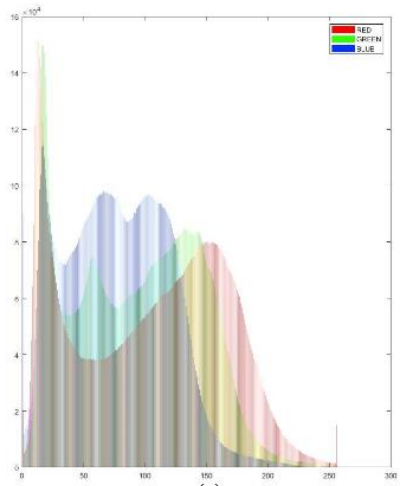

(a)

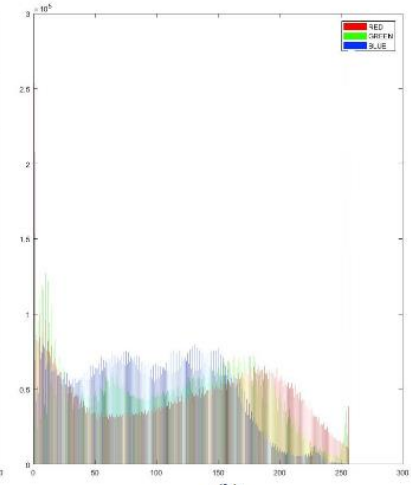

(b)
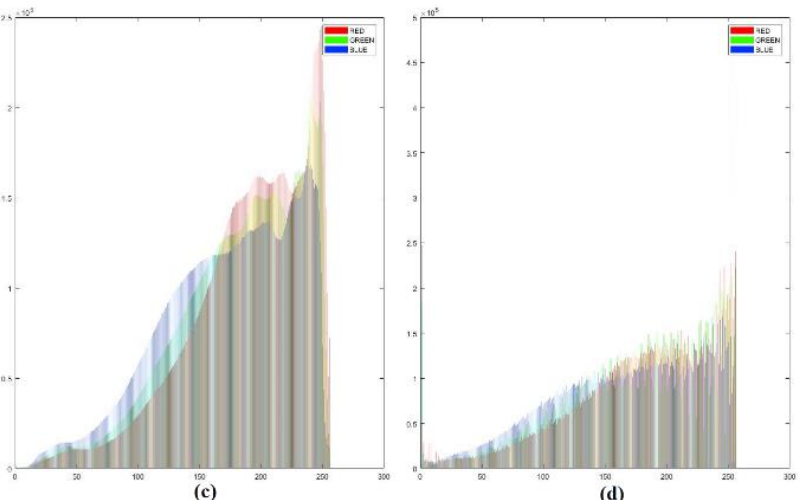

Figure 3: (a) Image I Histogram , (b) Image I Contrast Stretched Histogram, (c) Image II Histogram , (d) Image II Contrast Stretched Histogram

The performances of Weighted Differential Evolution (WDE) Algorithm, Artificial Bee Colony (ABC) Algorithm, histogram equalization and linear contrast stretching techniques were compared with the entropy value, edge density and sum of edge pixel 
count values. Experimental results are shown in Table 2.

Table 2: Experimental Results

\begin{tabular}{|c|c|c|c|c|c|c|}
\hline \multirow{2}{*}{ Methods } & \multicolumn{3}{|c|}{ Image I } & \multicolumn{3}{c|}{ Image II } \\
\cline { 2 - 7 } & $\begin{array}{c}\text { Entrop } \\
\mathrm{y}\end{array}$ & $\begin{array}{c}\text { Numbe } \\
\text { r of } \\
\text { edgels }\end{array}$ & $\begin{array}{c}\text { Mean } \\
\text { Edge } \\
\text { densit } \\
\mathrm{y}\end{array}$ & $\begin{array}{c}\text { Entrop } \\
\mathrm{y}\end{array}$ & $\begin{array}{c}\text { Numbe } \\
\text { r of } \\
\text { edgels }\end{array}$ & $\begin{array}{c}\text { Mean } \\
\text { Edge } \\
\text { densit } \\
\mathrm{y}\end{array}$ \\
\hline Original & $\begin{array}{c}7.5980 \\
6\end{array}$ & 393713 & $\begin{array}{c}0.3127 \\
7\end{array}$ & $\begin{array}{c}7.5059 \\
1\end{array}$ & 767146 & $\begin{array}{c}0.2656 \\
5\end{array}$ \\
\hline WDE & $\begin{array}{c}7.7962 \\
6\end{array}$ & 423634 & $\begin{array}{c}0.4260 \\
9\end{array}$ & $\begin{array}{c}7.9062 \\
4\end{array}$ & 789622 & $\begin{array}{c}0.2761 \\
5\end{array}$ \\
\hline ABC & $\begin{array}{c}7.7756 \\
9\end{array}$ & 422265 & $\begin{array}{c}0.4252 \\
7\end{array}$ & $\begin{array}{c}7.8768 \\
6\end{array}$ & 789316 & $\begin{array}{c}0.2702 \\
6\end{array}$ \\
\hline Hist. Eq. & $\begin{array}{c}7.7592 \\
4\end{array}$ & 396355 & $\begin{array}{c}0.3750 \\
3\end{array}$ & $\begin{array}{c}7.7250 \\
1\end{array}$ & 772215 & $\begin{array}{c}0.4121 \\
1\end{array}$ \\
\hline $\begin{array}{c}\text { Contrast } \\
\text { Stretchin }\end{array}$ & $\begin{array}{c}7.7648 \\
\text { g }\end{array}$ & 401916 & 0.3947 & 7.6788 & 770286 & 0.3135 \\
9
\end{tabular}

Comparison of the results in Table 2 shows that the heuristic algorithm approach gives better results than classical histogram equalization and contrast stretching methods. Enhanced images are shown in Figure 3 and Figure 4. Despite the use of Weighted Differential Evolution (WDE) Algorithm to solve different problems according to the literature, the image enhancement study was carried out for the first time. Compared to the well-known Artificial Bee Colony (ABC) Algorithm, the WDE algorithm has been found to be more successful. Because WDE uses completely random control parameters, it is more useful than other heuristic algorithms. WDE has no control parameters and since the control parameters are determined randomly, it has been proposed for the solution of image enhancement problems.

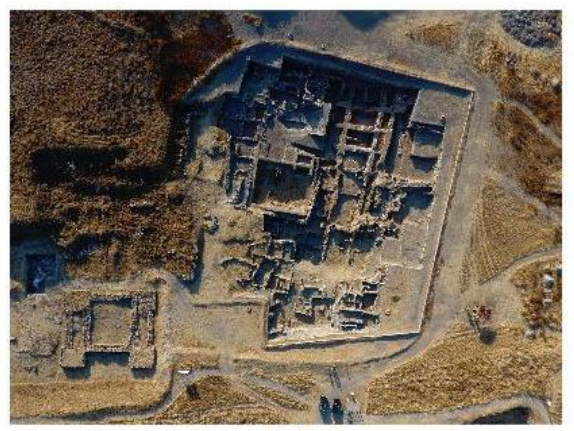

(a)

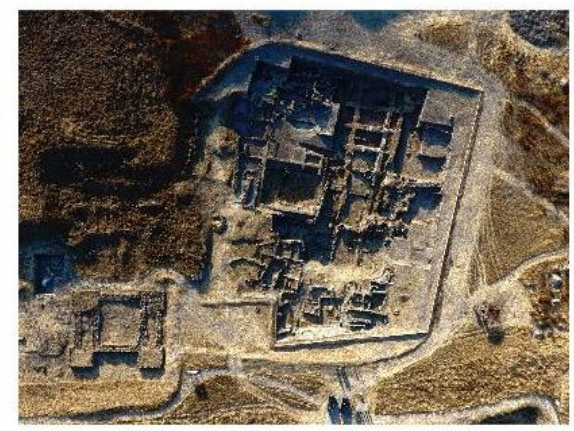

(b)

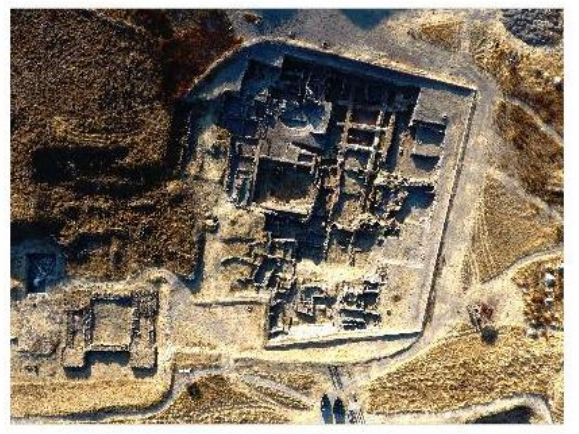

(c)

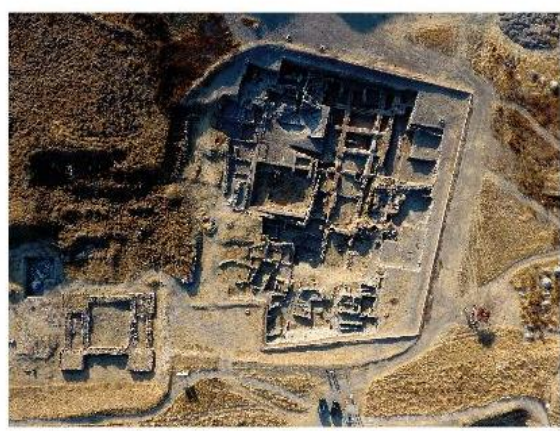

(d)

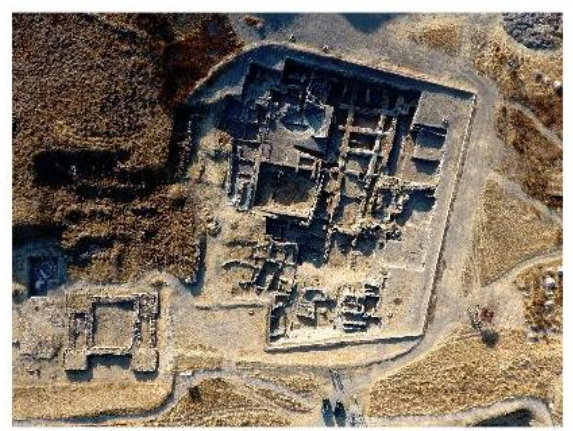

(e)

Figure 3: (a) Image_I (b) WDE (c) ABC (d) Histogram Equalization (e) Linear Contrast Stretching 


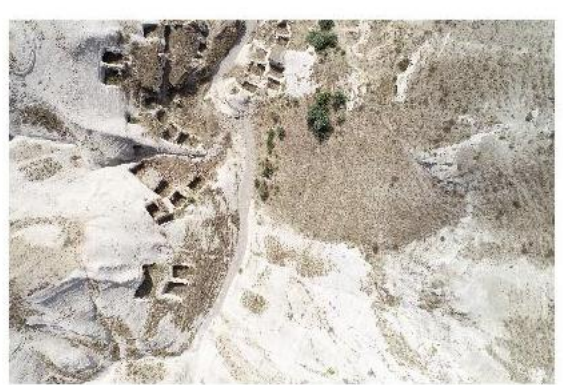

(a)

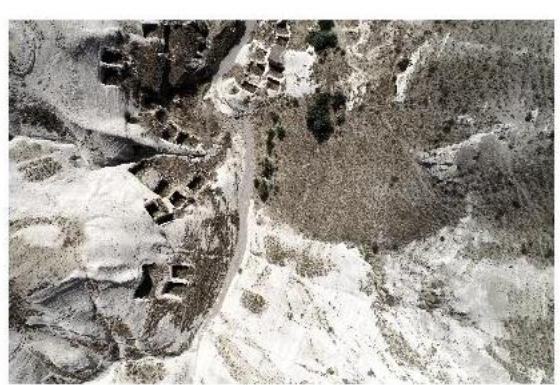

(b)

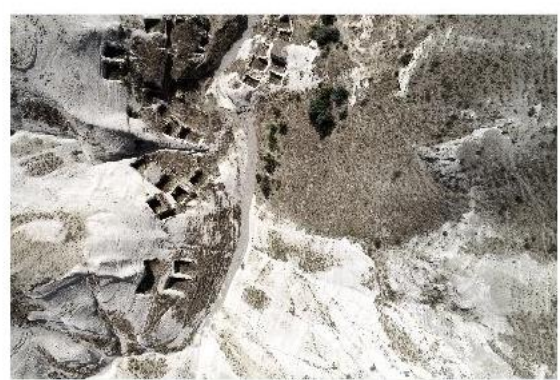

(c)

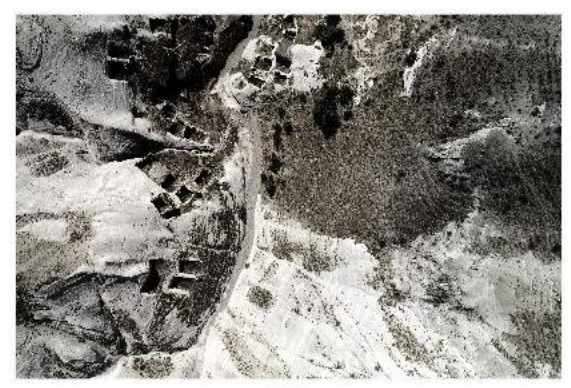

(d)

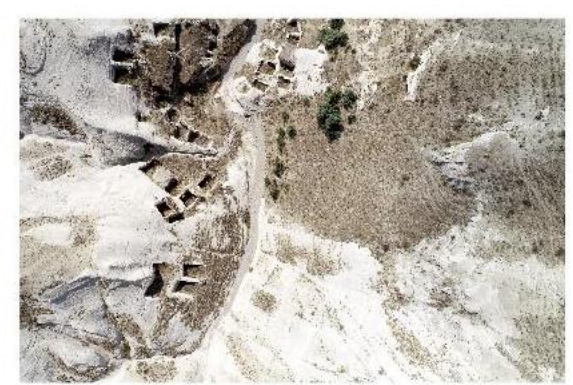

(e)

Figure 4: (a) Image_II (b) WDE (c) ABC (d) Histogram Equalization (e) Linear Contrast Stretching

\section{REFERENCES}

[1]. P. Burdziakowski, "Uav In Todays Photogrammetry-Application Areas And Challenges," International Multidisciplinary Scientific GeoConference: SGEM, vol. 18, no. 2.3, pp. 241-248, 2018.

[2]. M. Sauerbier and H. Eisenbeiss, "UAVs for the documentation of archaeological excavations," International Archives of the Photogrammetry, Remote Sensing and Spatial Information Sciences, vol. 38, no. 5, pp. 526-531, 2010.

[3]. M. Aljehani and M. Inoue, "Performance evaluation of multi-UAV system in postdisaster application: Validated by HITL simulator," IEEE Access, vol. 7, pp. 6438664400, 2019.

[4]. S. D'Oleire-Oltmanns, I. Marzolff, K. D. Peter, and J. B. Ries, "Unmanned aerial vehicle (UAV) for monitoring soil erosion in Morocco," Remote Sensing, vol. 4, no. 11, pp. 3390-3416, 2012.
[5]. B. Shi and C. Liu, "UAV for landslide mapping and deformation analysis," in International Conference on Intelligent Earth Observing and Applications 2015, 2015, vol. 9808, p. 98080P: International Society for Optics and Photonics.

[6]. A. Ulvï, "Analysis of the utility of the unmanned aerial vehicle (Uav) in volume calculation by using photogrammetric techniques," International Journal of Engineering and Geosciences, vol. 3, no. 2, pp. 43-49, 2018.

[7]. A. Rango and A. Laliberte, "Impact of flight regulations on effective use of unmanned aircraft systems for natural resources applications," Journal of Applied Remote Sensing, vol. 4, no. 1, p. 043539, 2010.

[8]. C. Amrullah, D. Suwardhi, and I. Meilano, "Product accuracy effect of oblique and vertical non-metric digital camera utilization in UAVphotogrammetry to determine fault plane," ISPRS Annals of the Photogrammetry, Remote Sensing and Spatial Information Sciences, vol. 3, p. 41, 2016. 
[9]. M. Kedzierski, D. Wierzbicki, A. Sekrecka, A. Fryskowska, P. Walczykowski, and J. Siewert, "Influence of lower atmosphere on the radiometric quality of unmanned aerial vehicle imagery," Remote Sensing, vol. 11, no. 10, p. 1214, 2019.

[10]. B. Chitradevi and P. Srimathi, "An overview on image processing techniques," International Journal of Innovative Research in Computer and Communication Engineering, vol. 2, no. 11, pp. 6466-6472, 2014.

[11]. R. Maini and H. Aggarwal, "A comprehensive review of image enhancement techniques," arXiv preprint arXiv:1003.4053, 2010.

[12]. S. M. Pizer et al., "Adaptive histogram equalization and its variations," Computer vision, graphics, and image processing, vol. 39, no. 3, pp. 355-368, 1987.

[13]. C.-C. Yang, "Image enhancement by modified contrast-stretching manipulation," Optics \& Laser Technology, vol. 38, no. 3, pp. 196-201, 2006.

[14]. A. Gorai and A. Ghosh, "Gray-level image enhancement by particle swarm optimization," in 2009 world congress on nature \& biologically inspired computing (NaBIC), 2009, pp. 72-77: IEEE.

[15]. S. S. Agaian, K. Panetta, and A. M. Grigoryan, "Transform-based image enhancement algorithms with performance measure," IEEE Transactions on image processing, vol. 10, no. 3, pp. 367-382, 2001.

[16]. J.-B. Martens and L. Meesters, "Image dissimilarity," Signal processing, vol. 70, no. 3, pp. 155-176, 1998.

[17]. A. S. Ashour, S. Samanta, N. Dey, N. Kausar, W. B. Abdessalemkaraa, and A. E. Hassanien, "Computed tomography image enhancement using cuckoo search: a log transform based approach," Journal of Signal and Information Processing, vol. 6, no. 03, p. 244, 2015.
[18]. M.-S. Shyu and J.-J. Leou, "A genetic algorithm approach to color image enhancement," Pattern Recognition, vol. 31, no. 7, pp. 871880, 1998.

[19]. L. dos Santos Coelho, J. G. Sauer, and M. Rudek, "Differential evolution optimization combined with chaotic sequences for image contrast enhancement," Chaos, solitons \& fractals, vol. 42, no. 1, pp. 522-529, 2009.

[20]. F. Katircioğlu and Z. Cingiz, "A Novel Gray Image Enhancement Using the Regional Similarity Transformation Function and Dragonfly Algorithm," El-Cezeri Journal of Science and Engineering, vol. 7, no. 3, pp. 1201-1219, 2020.

[21]. K. Gupta and A. Gupta, "Image enhancement using ant colony optimization," IOSR Journal of VLSI and Signal Processing, vol. 1, no. 3, pp. 38-45, 2012.

[22]. Z. Ye, M. Wang, Z. Hu, and W. Liu, "An adaptive image enhancement technique by combining cuckoo search and particle swarm optimization algorithm," Computational intelligence and neuroscience, vol. 2015, 2015.

[23]. P. Civicioglu, E. Besdok, M. A. Gunen, and U. H. Atasever, "Weighted differential evolution algorithm for numerical function optimization: a comparative study with cuckoo search, artificial bee colony, adaptive differential evolution, and backtracking search optimization algorithms," Neural Computing and Applications, vol. 32, no. 8, pp. 3923-3937, 2020.

[24]. M. A. Gunen, E. Besdok, P. Civicioglu, and U. H. Atasever, "Camera calibration by using weighted differential evolution algorithm: a comparative study with ABC, PSO, COBIDE, DE, CS, GWO, TLBO, MVMO, FOA, LSHADE, ZHANG and BOUGUET," Neural Computing \& Applications, 2020. 
[25]. P. Civicioglu and E. Besdok, "Bernstain-search differential evolution algorithm for numerical function optimization," Expert Systems with Applications, vol. 138, p. 112831, 2019.

[26]. D. H. Choi, I. H. Jang, M. H. Kim, and N. C. Kim, "Color image enhancement using singlescale retinex based on an improved image formation model," in 2008 16th European Signal Processing Conference, 2008, pp. 1-5: IEEE.

[27]. D. Ghimire and J. Lee, "Color image enhancement in HSV space using nonlinear transfer function and neighborhood dependent approach with preserving details," in 2010 Fourth Pacific-Rim Symposium on Image and Video Technology, 2010, pp. 422-426: IEEE.

[28]. S. K. Naik and C. Murthy, "Hue-preserving color image enhancement without gamut problem," IEEE Transactions on image processing, vol. 12, no. 12, pp. 1591-1598, 2003.

[29]. C. C. Yang and S. H. Kwok, "Efficient gamut clipping for color image processing using LHS and YIQ," Optical Engineering, vol. 42, no. 3, pp. 701-711, 2003.

[30]. M. Nikolova and G. Steidl, "Fast hue and range preserving histogram specification: Theory and new algorithms for color image enhancement," IEEE transactions on image processing, vol. 23, no. 9, pp. 4087-4100, 2014.

[31]. X. Su, W. Fang, Q. Shen, and X. Hao, "An image enhancement method using the quantum-behaved particle swarm optimization with an adaptive strategy," Mathematical Problems in Engineering, vol. 2013, 2013.

[32]. P. Civicioglu and E. Besdok, "A conceptual comparison of the Cuckoo-search, particle swarm optimization, differential evolution and artificial bee colony algorithms," Artificial intelligence review, vol. 39, no. 4, pp. 315-346, 2013.
[33]. P. Civicioglu and E. Besdok, "A+ Evolutionary search algorithm and QR decomposition based rotation invariant crossover operator," Expert Systems with Applications, vol. 103, pp. 49-62, 2018.

[34]. T. Kurban, P. Civicioglu, R. Kurban, and E. Besdok, "Comparison of evolutionary and swarm based computational techniques for multilevel color image thresholding," Applied Soft Computing, vol. 23, pp. 128-143, 2014.

[35]. D. Karaboga and B. Basturk, "On the performance of artificial bee colony (ABC) algorithm," Applied soft computing, vol. 8, no. 1, pp. 687-697, 2008.

[36]. D. Karaboga and B. Basturk, "A powerful and efficient algorithm for numerical function optimization: artificial bee colony (ABC) algorithm," Journal of global optimization, vol. 39, no. 3, pp. 459-471, 2007.

[37]. D. Karaboga, B. Gorkemli, C. Ozturk, and N. Karaboga, "A comprehensive survey: artificial bee colony (ABC) algorithm and applications," Artificial Intelligence Review, vol. 42, no. 1, pp. 21-57, 2014.

[38]. D. Karaboga and C. Ozturk, "A novel clustering approach: Artificial Bee Colony (ABC) algorithm," Applied soft computing, vol. 11, no. 1, pp. 652-657, 2011.

[39]. C. Munteanu and A. Rosa, "Towards automatic image enhancement using genetic algorithms," in Proceedings of the 2000 Congress on Evolutionary Computation. CEC00 (Cat. No. 00TH8512), 2000, vol. 2, pp. 1535-1542: IEEE.

[40]. A. Draa and A. Bouaziz, "An artificial bee colony algorithm for image contrast enhancement," Swarm and Evolutionary computation, vol. 16, pp. 69-84, 2014.

[41]. J.-H. Han, S. Yang, and B.-U. Lee, "A novel 3-D color histogram equalization method with uniform 1-D gray scale histogram," IEEE 
Transactions on Image Processing, vol. 20, no.

2, pp. 506-512, 2010.

[42]. M. Veluchamy and B. Subramani, "Image contrast and color enhancement using adaptive gamma correction and histogram equalization," Optik, vol. 183, pp. 329-337, 2019.

\section{Cite this article as :}

Ahmet Emin Karkınll, Abdüsselam Kesikoğlu, "Enhancement of UAV-Aerial Images Using Weighted Differential Evolution Algorithm", International Journal of Scientific Research in Computer Science, Engineering and Information Technology (IJSRCSEIT), ISSN : 2456-3307, Volume 7 Issue 2, pp. 196-206, March-April 2021. Available at doi : https://doi.org/10.32628/CSEIT217248 Journal URL : https://ijsrcseit.com/CSEIT217248 